\title{
Expression of foreign aquaporin genes in lily pollen protoplasts
}

\author{
Keiji Yamada ${ }^{1}$, Ken-ichi Yanada ${ }^{2}$, Atsushi Matsuzawa ${ }^{1}$, Ichiro Tanaka ${ }^{1,2}$, \\ Hajime Shiota ${ }^{1,2, *}$ \\ ${ }^{1}$ International Graduate School of Arts and Sciences, Yokohama City University, Yokohama, Kanagawa 236-0027, \\ Japan; ${ }^{2}$ Graduate School of Nanobioscience, Yokohama City University, Yokohama, Kanagawa 236-0027, Japan \\ *E-mail: hshiota@yokohama-cu.ac.jpＴel: +81-45-787-2318 Fax: +81-45-787-2413
}

Received May 9, 2011; accepted September 22, 2011 (Edited by Y. Ozeki)

\begin{abstract}
Aquaporins facilitate water flux across biomembranes in cells and are involved in various physiological phenomena in several plant tissues. Generally, the water-flux activity of exogenously expressed aquaporins is measured in Xenopus laevis oocytes or yeast. However, heterogeneous systems are not likely to be optimal for plant aquaporin analysis. Thus, we created a new experimental system for functional analysis of plant aquaporins using lily (Lilium longiflorum) pollen protoplasts. Large protoplasts with uniform diameters of approximately $95 \mu \mathrm{m}$ were isolated from lily pollen grains. No plasma membrane intrinsic protein (PIP) aquaporin was detected in lily pollen. For ectopic expression of PIPs in the lily pollen protoplasts, we constructed plasmids in which Arabidopsis AtPIP1;1 or AtPIP2;1 was under the control of a strong pollen-specific promoter (maize $Z m 13$ ). The PCR-amplified DNA fragments were transformed into the pollen protoplasts by electroporation. Between 45 and $60 \%$ of protoplasts were successfully transformed. The protoplasts expressing AtPIP2;1 significantly increased in volume in a hypotonic solution $(350 \mathrm{mM}$ mannitol), compared with the vector control. In contrast, the changes in volume of the protoplasts expressing AtPIP1;1 were similar to that of the vector control. This result suggests that PIP2 induces higher water-flux activity in plant cells, whereas PIP1 does not. Thus, we propose the lily pollen protoplast as a simple and useful experimental system to analyze the function of plant aquaporins.
\end{abstract}

Key words: Lilium longiflorum, plasma membrane aquaporin, pollen, protoplast, water permeability.

Water flux across biomembranes is regulated by membrane protein aquaporins (Gomes et al. 2009). Aquaporins are present in the plasma membrane or tonoplast and function as a water channel, forming a homotetramer or heterotetramer. Genome projects have revealed that more than 30 aquaporins exist in plants. Plant aquaporins are classified into plasma membrane intrinsic proteins (PIPs), tonoplast intrinsic proteins (TIPs), small basic intrinsic proteins (SIPs), and Nod26like intrinsic proteins (NIPs) (Gomes et al. 2009). Furthermore, PIPs are divided into two subgroups, PIP1 and PIP2. In plants, the various aquaporins are likely involved in the maintenance of adequate water levels in various tissues at different developmental stages.

Generally, functional analysis of aquaporins is performed using Xenopus laevis oocytes and yeast cells. Xenopus oocytes microinjected with complementary aquaporin RNAs are incubated in a hypotonic solution, and the water-flux activity is calculated from the cell volume increase during incubation (Preston et al. 1992). The increased cell volume of yeast (Saccharomyces cerevisiae strain BJ5458) transformed with aquaporin genes is measured during incubation in the hypotonic solution using a stopped-flow light-scattering spectrometer (Suga and Maeshima 2004). For plant aquaporins, however, these heterogeneous experimental systems are not sufficiently precise for functional analysis. The mechanisms of protein folding and trafficking to the plasma membrane may differ between plant and animal cells, or unknown factors that interact with plant aquaporins may be absent in animal cells (Fetter et al. 2004; Grote et al. 1999; Suga and Maeshima 2004). Therefore, a functional analysis system using plant cells is necessary.

In plants, the protoplast volume change during incubation in a hypotonic solution is measured by analysis of osmotic water permeability (Grote et al. 1999; Murai-Hatano and Kuwagata 2007; Suga et al. 2003). However, it is difficult to correctly measure the size of protoplasts because they are small and nonuniform. Furthermore, in protoplasts expressing exogenous aquaporins, the activities of both endogenous and exogenous aquaporins have been observed. In lily (Lilium longiflorum), large pollen grains are produced and large protoplasts can be isolated from pollen grains (Tanaka et al. 1987). Synchronized developmental stages

Abbreviations: CCD, charge-coupled device; GFP, green fluorescent protein; ORF, open reading frame; PIP, plasma membrane intrinsic protein; PVDF, polyvinylidene fluoride

This article can be found at http://www.jspcmb.jp/

Published online November 30, 2011 
of pollen can be obtained by using flower buds of the same length (Tanaka et al. 1987). Lily pollen protoplasts have been widely used for investigating the proton pump, potassium channel, cytosolic calcium, or osmotic water permeability (Gehwolf et al. 2002; Griessner and Obermeyer 2003; Shang et al. 2005; Sommer et al. 2007). For gene transfer, electroporation and polyethylene glycol-mediated transformation can be used for lily pollen protoplasts, whereas particle bombardment is typically used for lily pollen grains (Miyoshi et al. 1995; Nishihara et al. 1993). In this study, we propose a novel, convenient experimental system for functional analysis of plant aquaporins, exploiting the advantages of lily pollen protoplasts.

Lily pollen protoplasts were isolated using the method described by Tanaka et al. (1987) with modifications. Lily (Lilium longiflorum Thunb. cv. Hinomoto) flower buds were obtained from plants grown in a greenhouse. The nearly mature pollen grains were isolated from anthers in flower buds $150-170 \mathrm{~mm}$ in length and incubated in a pollen protoplast enzyme solution $(1.0 \%$ Macerozyme R-10, 1.0\% cellulase Onozuka R-10, 0.5\% potassium dextran sulfate, $0.5 \mathrm{M}$ sucrose, White's mineral salts and vitamins, $\mathrm{pH} 5.8$ ) at $30^{\circ} \mathrm{C}$ for $2 \mathrm{~h}$ with gentle reciprocal shaking. After enzyme treatment, protoplasts were washed three times with a pollen protoplast culture medium $(0.5 \mathrm{M}$ sucrose, White's mineral salts and vitamins, $\mathrm{pH} 5.8$ ) by centrifugation at $600 \times g$ for $5 \mathrm{~min}$. The final suspension was layered on culture medium containing $15 \%$ Percoll and then centrifuged at $600 \times g$ for $5 \mathrm{~min}$. After centrifugation, the pollen protoplasts separated on the Percoll density gradient were collected and suspended in the culture medium.

The protoplasts isolated from lily pollen grains were completely globular and their protoplasm were uniform (Supplementary Figure S1). The protoplasts were large in size and the relative diameters of the protoplasts were within a very narrow range [average diameter of $94.5 \pm 1.5 \mu \mathrm{m}(n=10)]$. Because protoplasts from plant tissues are generally small, specific experimental devices (e.g., micromanipulators) are required for handling them (Murai-Hatano and Kuwagata 2007; Suga et al. 2003). Conversely, lily pollen protoplasts are easily handled using a micropipette because they are usually large in size. When lily pollen protoplasts are isolated from synchronized developmental stages of pollen grains, each lily pollen protoplast has uniform characteristics (Tanaka et al. 1987).

To detect plasma membrane aquaporins (PIPs) in lily pollen, Western blot analysis was performed. Plasma membrane fractions containing membrane proteins were extracted from leaves, anther walls, and nearly mature lily pollen grains using an aqueous two-polymer phase system (Suga et al. 2001; Yoshida et al. 1983). Plasma membranes were fractionated by sodium dodecyl sulfate-polyacrylamide gel (12\%) electrophoresis and transferred to a polyvinylidene fluoride (PVDF) membrane (Hybond-P, GE Healthcare, Piscataway, NJ, USA). Western blot analysis was performed using a primary antibody against radish PIP1 (anti-PAQs antibody) that recognizes both PIP1 and PIP2 (Ohshima et al. 2001; Sakurai et al. 2005; Suga et al. 2001). Signals were detected using an ECL plus system (GE Healthcare) and visualized with X-ray film (Hyper Film ECL, GE Healthcare). Bands of 28 and $56 \mathrm{kDa}$ were detected in lily leaves and anther walls (Figure 1), likely representing PIP monomers and dimers, respectively. Alternatively, no signal was detected in pollen grains, despite the use of a double volume plasma membrane fraction (Figure 1). This suggests that PIPs exist in lily leaves and anther walls, but not in pollen grains. In pollen grains, water uptake is likely caused by water permeability through the plasma membrane lipid bilayers without aquaporins, as seen in Xenopus oocytes (Sommer et al. 2008). Therefore, foreign aquaporins transfected into the protoplasts could affect osmotic water permeability.

The plasmids for gene transfer were constructed from pBI221 (Clontech, Polo Alto, CA, USA). To monitor

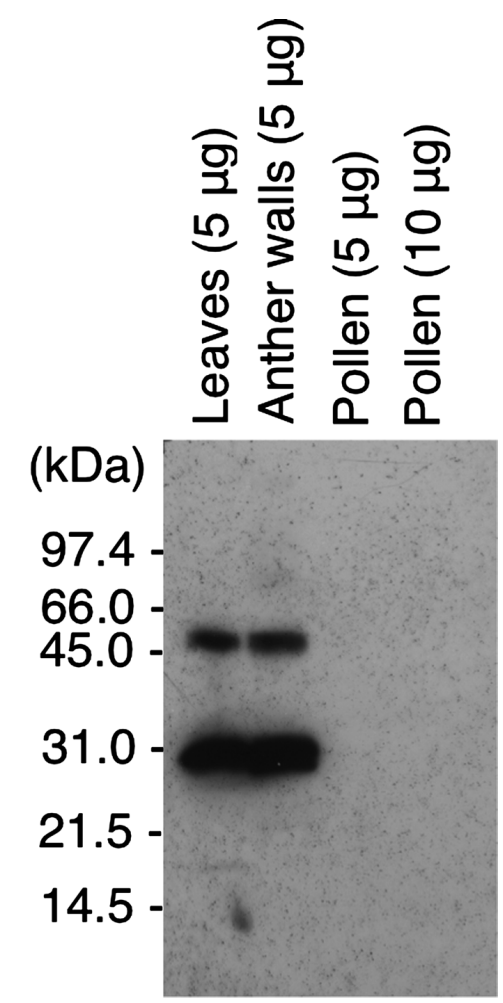

Figure 1. Immunological detection of PIPs in lily tissues. Plasma membrane fractions containing membrane proteins extracted from leaves, anther walls, and pollen grains were separated by SDS-PAGE (12\%), electroblotted, and immunostained with purified anti-PIPs immunoglobulins. Signals were detected by enhanced chemiluminescence followed by exposure to X-ray film. 
gene transfer into pollen protoplasts, the genes for green fluorescent protein (GFP) (Marshall et al. 1995) or red fluorescent protein (DsRed2, Clontech) (Nahalkova and Fatehi 2003) were placed under control of a strong pollen-specific Zm13 promoter (Hamilton et al. 1989) (pZm13::GFP, pZm13::DsRed2) (Figure 2A, B). To express both aquaporin and reporter genes under control of the Zm13 promoter, the plasmid pZm13::AtPIP1;1 was constructed to express Arabidopsis AtPIP1;1 (Accession no. AK229222) (Jang et al. 2004), based on pZm13::GFP (Figure 2C). The pZm13::AtPIP2;1, expressing Arabidopsis AtPIP2;1 (Accession no. AY072374) (Kammerloher et al. 1994), was constructed based on pZm13::DsRed2 (Figure 2D).

For transformation of pollen protoplasts, PCRamplified DNA fragments were prepared. PCR was performed using a specific primer set [PS1 (5'-GTTTTCCCAGTCACGAC-3') and PA1 (5'-GCCGATTCATTAATGCAG- $\left.3^{\prime}\right)$ ], the plasmids discussed above as templates, and Ex Taq DNA polymerase (Takara Bio, Ohtsu, Japan) under the following temperature conditions: 40 cycles of $94^{\circ} \mathrm{C}$ for $30 \mathrm{~s}, 52^{\circ} \mathrm{C}$ for $30 \mathrm{~s}$, and $72^{\circ} \mathrm{C}$ for 2 or $4 \mathrm{~min}$. The DNA fragments were extracted directly and purified using phenol/chloroform extraction followed by ethanol precipitation. For gene transfer into lily pollen protoplasts, electroporation was performed as described by Miyoshi et al. (1995) with modifications. The isolated protoplasts were suspended in electroporation buffer ( $3 \mathrm{mM}$ MES, $42 \mathrm{mM} \mathrm{KCl,} 4 \mathrm{mM}$ $\mathrm{CaCl}_{2}, 0.4 \mathrm{M}$ mannitol, $\mathrm{pH} 5.8$ ) at a density of $1 \times 10^{5}$ protoplasts $\mathrm{ml}^{-1}$. The protoplast suspension $(450 \mu \mathrm{l})$ and $100 \mu \mathrm{g} \mathrm{ml}^{-1}$ of DNA solution $(50 \mu \mathrm{l})$ were placed on ice for $10 \mathrm{~min}$. The mixture was transferred into an ice-cold (10 min cool time) electroporation cuvette (165-2088, Bio-Rad, Richmond, CA, USA). A single pulse at a field strength of $500 \mathrm{~V} \mathrm{~cm}^{-1}$ with a condenser capacitance of



Figure 2. Plasmid construction. Plasmids for gene transfer into lily pollen protoplasts were constructed. (A) pZm13::GFP, (B) pZm13::DsRed2, (C) pZm13::AtPIP1;1, and (D) pZm13::AtPIP2;1. The plasmids were used as templates for PCR, and amplified DNA fragments were used for transformation. Arrows show the positions of the PCR primers.
$250 \mu \mathrm{F}$ was delivered to the protoplast suspension in a 0.5-ml Gene Pulser electroporation chamber (Bio-Rad). The suspension was immediately cooled on ice for 10 min and then incubated at $25^{\circ} \mathrm{C}$ for $10 \mathrm{~min}$. After incubation, the protoplasts were washed with pollen protoplast culture medium and suspended in a 5- to 10times volume of pollen protoplast enzyme solution. The suspension was transferred into a Petri dish $(60 \mathrm{~mm}$ in diameter) and incubated at $25^{\circ} \mathrm{C}$ in darkness for $20-24 \mathrm{~h}$.

After $20-24 \mathrm{~h}$ of culturing, transformed protoplasts exhibited green or red fluorescence (Supplementary Figure S2). The gene transfer efficiency was high (53.4\%, the average of three individual experiments) in the case of $\mathrm{Zm} 13:: \mathrm{GFP}$, as also reported by Miyoshi et al. (1995) (60-70\%). In lily pollen, gene transfer efficiency by electroporation is higher than that by particle bombardment, which results in less than $1 \%$ gene transfer efficiency (Miyoshi et al. 1995; Nishihara et al. 1993). This experimental system obtained a large number of transfected protoplasts because of the high (45-60\%) gene transfer efficiency attained using electroporation.

Lily pollen protoplasts reconstructed their cell walls during the 24-h incubation period (Miki-Hiroshige et al. 1988). The volume increase of protoplasts with reconstructed cell walls was likely physically limited in the hypotonic solution. After electroporation, the protoplasts were incubated in a pollen protoplast enzyme solution to prevent reconstruction of cell walls. After incubation in the enzyme solution for $20-24 \mathrm{~h}$, the transformed protoplasts exhibited diameters of 100.0$101.1 \mu \mathrm{m}$ (Figure 3 ). In all cases, the relative protoplast diameters were within a narrow range (Figure 3). In addition, cell walls were not reconstructed and the protoplasts expanded successfully in the hypotonic solution (Supplementary Figure S2). In our experimental system, the protoplast swelling assay was successful both before and after electroporation, indicating that the electroporation and subsequent culturing caused only slight damage to the protoplasts.

Protoplasts displaying stronger green (GFP) or red (DsRed2) fluorescence (Supplementary Figure S2), which expressed both aquaporin and reporter genes, were selected with $0.5 \mu \mathrm{l}$ of medium using a micropipette and transferred from an isotonic solution (500 $\mathrm{mM}$ mannitol) into $50 \mu \mathrm{l}$ of a hypotonic solution $(350 \mathrm{mM}$ mannitol) prepared in a ground cavity (14 to $15 \mathrm{~mm}$ diameter and 0.4-mm depth) on a microscope slide. Micrographs of protoplasts in solution were collected at 30-s intervals, and the diameters and volumes of protoplasts were measured using the CCD camera system (VB7010, Keyence, Osaka, Japan). The relative volume of protoplasts was calculated as the protoplast volume at each period/protoplast volume at the beginning of observation ( $30 \mathrm{~s}$ after transferring). To compare more 


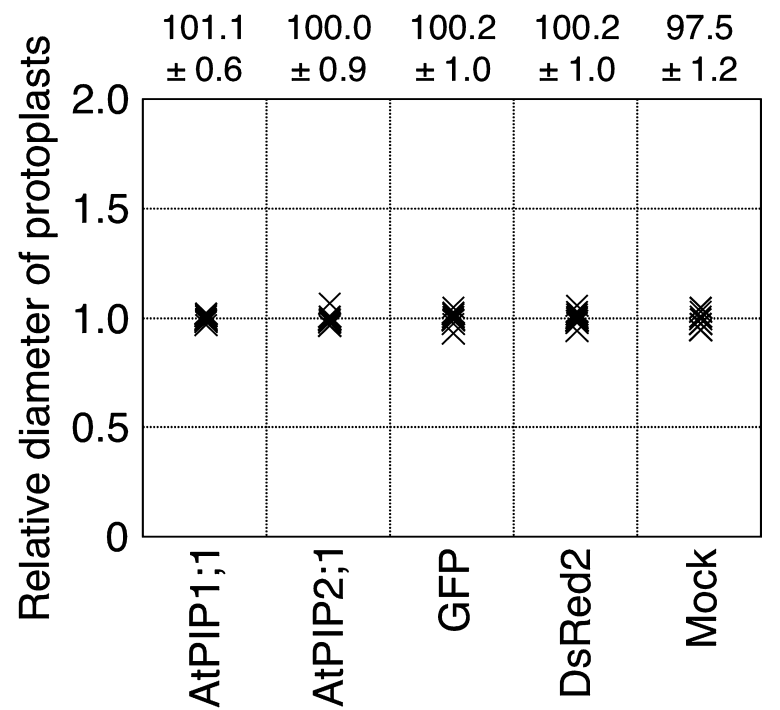

Figure 3. Relative diameters of transfected pollen protoplasts. The relative diameters of lily pollen protoplasts transfected with Zm13::AtPIP1;1 (AtPIP1;1), Zm13::AtPIP2;1 (AtPIP2;1), Zm13::GFP (GFP), Zm13::DsRed2 (DsRed2), and no DNA fragments (mock) are shown. The average diameters of ten protoplasts are shown as 1.0. The average diameters $(\mu \mathrm{m})$ of the protoplasts are shown above the graph $(n=10, \pm \mathrm{SE})$.

explicitly the change in protoplast expansion during incubation, the change in relative volume of protoplasts was exhibited (Murai-Hatano and Kuwagata 2007; Sommer et al. 2007; Suga et al. 2003).

After transferring to the hypotonic solution, the relative volume of lily pollen protoplasts increased. At $600 \mathrm{~s}$ after transferring to hypotonic solution, the values of relative volume of pollen protoplasts were stable and slightly different between Figure $4 \mathrm{~A}$ and $4 \mathrm{~B}$. The difference may be caused by the variety of strength and permeability of plasma membrane in lily pollen, because they are affected by pollen development or cultivation condition (Tanaka et al. 1987). The increase in the relative volume of the protoplasts transfected with Zm13::AtPIP1;1 was very similar to that of the protoplasts transfected with $\mathrm{Zm13}:$ GFP (as a control group) (Figure 4A). The increase in the relative volume of the protoplasts transfected with Zm13::AtPIP2;1 was faster than that of the protoplasts transfected with Zm13::DsRed2 (control group) (Figure 4B). Thus, AtPIP2;1 induced high osmotic water permeability, whereas AtPIP1;1 induced very low osmotic water permeability in lily pollen protoplasts. In Xenopus oocytes or yeast cells, it is generally believed that PIP2 alone plays a role in water permeability, but PIP1 alone has no function (Fetter et al. 2004; Kammerloher et al. 1994; Suga and Maeshima 2004). Zelazny et al. (2007) also reported that in maize mesophyll protoplasts, transfected ZmPIP1s were localized and accumulated in the internal structures around the nucleus, within the cytosol and near the plasma membrane, but not in the
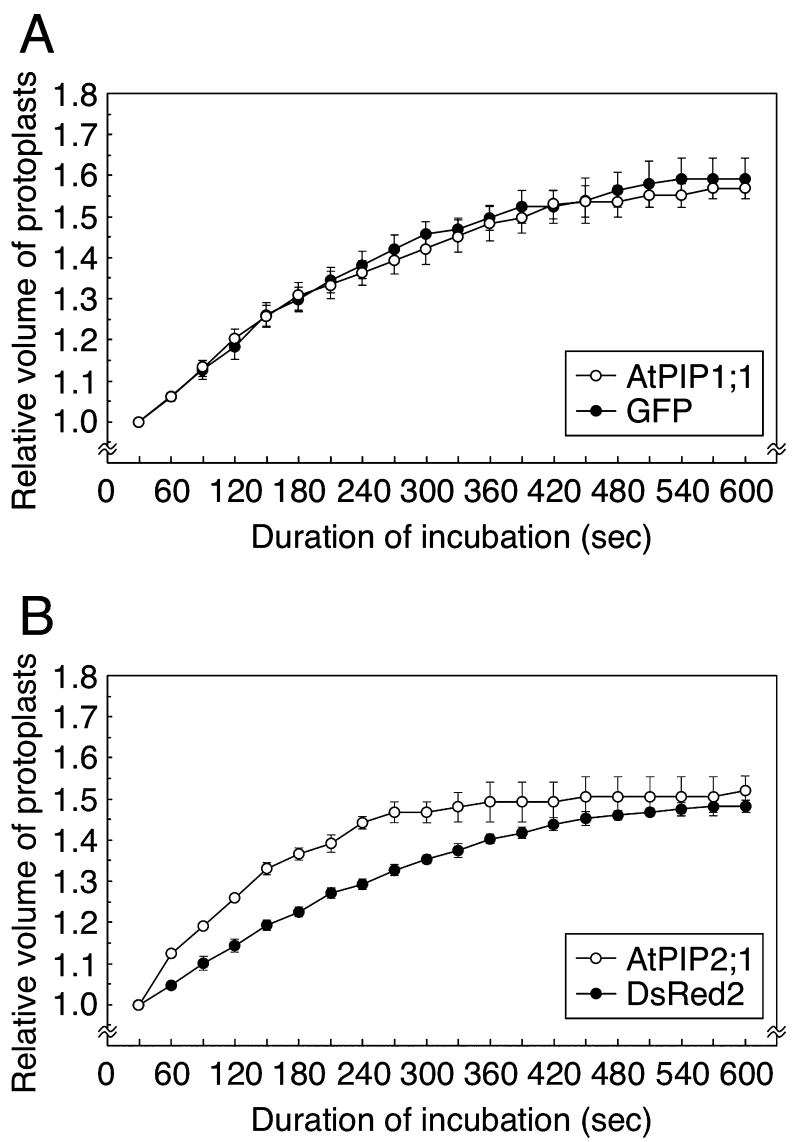

Figure 4. Change in volume of transfected pollen protoplasts in a hypotonic solution. The change in the relative volume of transfected pollen protoplasts is shown. The relative volume of protoplasts was calculated as the protoplast volume at each period/protoplast volume at the start of observation ( $30 \mathrm{~s}$ after transferring). Protoplasts transfected with Zm13::AtPIP1;1 (A) and Zm13::AtPIP2;1 (B). The values show the average $(n=3-5, \pm \mathrm{SE})$.

plasma membrane. In our system, it is likely that the solely expressed AtPIP1;1 did not induce water permeability because they were improperly trafficked to the plasma membrane.

The intensity of GFP or DsRed2 fluorescence was different among individual protoplasts (Supplementary Figure S3). This suggests that the expression level of transfected aquaporins may differ in each protoplast. Using the protoplasts showing stronger fluorescence, the change in relative protoplast volume remained stable in the hypotonic solution (Figure 4). In the lily system, correlation of expression level with the reporter and aquaporin concentrations could not be confirmed. Therefore, the pollen protoplast system is suited for the qualitative aquaporin function assay, whereas the Xenopus oocyte system, with microinjection of complementary RNAs, is desirable for quantitative analysis.

We propose that the pollen protoplast system can be used as a simple experimental tool to analyze the function (osmotic water permeability) of plant 
aquaporins. Sommer et al. (2008) reported the water permeability of Arabidopsis aquaporins in protoplasts from lily pollen grains and pollen tubes using an experimental method similar to our system. In the method reported by Sommer et al. (2008), transfected protoplasts were isolated from pollen grains after particle bombardment, and the tomato LAT52 promoter was used as a pollen-specific promoter for gene expression in pollen protoplasts. In contrast, in our system, gene transfer by electroporation was performed after protoplast isolation, and the $Z m 13$ promoter was used for gene expression in pollen protoplasts. Electroporation into isolated pollen protoplasts is more efficient than particle bombardment followed by protoplast isolation because the gene transfer efficiency of particle bombardment is much lower (Miyoshi et al. 1995; Nishihara et al. 1993). Furthermore, many transfected protoplasts are lost during protoplast isolation from pollen grains. Miyoshi et al. (1995) reported that the Zm13 promoter showed higher activity than the LAT52 promoter in lily pollen as a monocotyledonous plant. In our system, it was possible to maintain a high level of aquaporin expression and to observe changes in osmotic water permeability using the strong $Z m 13$ promoter. Therefore, our experimental system is simpler and more efficient than the system described by Sommer et al. (2008).

In this study, we propose a more convenient and effective experimental system for functional analysis of plant aquaporins using lily pollen protoplasts as plant cells. We believe that the function of several plant aquaporins can be determined using this experimental system.

\section{Acknowledgements}

We thank Prof. M. Maeshima of Nagoya University for the generous gift of antibodies against plant PIPs. We also thank Dr. Y. Niwa of the University of Shizuoka for the generous gift of a GFP gene. This work was supported by a Grant in Support of the Promotion of Research at Yokohama City University.

\section{References}

Fetter K, Van Wilder V, Moshelion M, Chaumont F (2004) Interactions between plasma membrane aquaporins modulate their water channel activity. Plant Cell 16: 215-228

Gehwolf R, Griessner M, Pertl H, Obermeyer G (2002) First patch, then catch: measuring the activity and the mRNA transcripts of a proton pump in individual Lilium pollen protoplasts. FEBS Lett 512: 152-156

Gomes D, Agasse A, Thiébaud P, Delrot S, Gerós H, Chaumont F (2009) Aquaporins are multifunctional water and solute transporters highly divergent in living organisms. Biochim Biophys Acta 1788: 1213-1228

Griessner M, Obermeyer G (2003) Characterization of whole-cell $\mathrm{K}^{+}$currents across the plasma membrane of pollen grain and tube protoplasts of Lilium longiflorum. J Membr Biol 193: 99-108

Grote K, Gimmler H, Kaidenhoff R (1999) Coulter counter cell size determination of protoplasts from Arabidopsis thaliana PIPlb aquaporin antisense lines under iso- and hypo-osmotic conditions. Protoplasma 210: 31-35

Hamilton DA, Bashe DM, Stinton JR, Mascarenhas JP (1989) Characterization of a pollen-specific genomic clone from maize. Sex Plant Reprod 2: 208-212

Jang JY, Kim DG, Kim YO, Kim JS, Kang H (2004) An expression analysis of a gene family encoding plasma membrane aquaporins in response to abiotic stresses in Arabidopsis thaliana. Plant Mol Biol 54: 713-725

Kammerloher W, Fischer U, Piechottka GP, Schiiffner AR (1994) Water channels in the plant plasma membrane cloned by immunoselection from a mammalian expression system. Plant J 6: 187-199

Marshall J, Molloy R, Moss GW, Howe JR, Hughes TE (1995) The jellyfish green fluorescent protein: a new tool for studying ion channel expression and function. Neuron 14: 211-215

Miki-Hiroshige H, Nakamura S, Tanaka I (1988) Ultrastructural research on cell wall regeneration by cultured pollen protoplasts of Lilium longiflorum. Sex Plant Reprod 1: 36-45

Miyoshi H, Usami T, Tanaka I (1995) High level of GUS gene expression driven by pollen-specific promoters in electroporated lily pollen protoplasts. Sex Plant Reprod 8: 205-209

Murai-Hatano M, Kuwagata T (2007) Osmotic water permeability of plasma and vacuolar membranes in protoplasts I. High osmotic water permeability in radish (Raphanus sativus) root cells as measured by a new method. J Plant Res 120: 175-189

Nahalkova J, Fatehi J (2003) Red fluorescent protein (DsRed2) as a novel reporter in Fusarium oxysporum f. sp. lycopersici. FEMS Microbiol Lett 225: 305-309

Nishihara M, Ito M, Tanaka I, Kyo M, Ono K, Irifune K, Morikawa $H$ (1993) Expression of the $\beta$-glucuronidase gene in pollen of lily (Lilium longiflorum), tobacco (Nicotiana tabacum), Nicotiana rustica and peony (Paeonia lactiflora) by particle bombardment. Plant Physiol 102: 357-361

Ohshima Y, Iwasaki I, Suga S, Murakami M, Inoue K, Maeshima M (2001) Low aquaporin content and low osmotic water permeability of the plasma and vacuolar membranes of a CAM plant Graptopetalum paraguayense: comparison with radish. Plant Cell Physiol 42: 1119-1129

Preston GM, Carroll TP, Guggino WB, Agre P (1992) Appearance of water channels in Xenopus oocytes expressing red cell CHIP28 protein. Science 256: 385-387

Sakurai J, Ishikawa F, Yamaguchi T, Uemura M, Maeshima M (2005) Identification of 33 rice aquaporin genes and analysis of their expression and function. Plant Cell Physiol 46: 1568-1577

Shang ZL, Ma LG, Zhang HL, He RR, Wang XC, Cui SJ, Sun DY (2005) $\mathrm{Ca}^{2+}$ influx into lily pollen grains through a hyperpolarization-activated $\mathrm{Ca}^{2+}$-permeable channel which can be regulated by extracellular CaM. Plant Cell Physiol 46: 598-608

Sommer A, Mahlknecht G, Obermeyer G (2007) Measuring the osmotic water permeability of the plant protoplast plasma membrane: implication of the nonosmotic volume. $J \mathrm{Membr}$ Biol 215: 111-123

Sommer A, Geist B, Ines OD, Gehwolf R, Schäffner AR, Obermeyer G (2008) Ectopic expression of Arabidopsis thaliana plasma membrane intrinsic protein 2 aquaporins in lily pollen 
increases the plasma membrane water permeability of grain but not of tube protoplasts. New Phytol 180: 787-797

Suga S, Maeshima M (2004) Water channel activity of radish plasma membrane aquaporins heterologously expressed in yeast and their modification by site-directed mutagenesis. Plant Cell Physiol 45: 823-830

Suga S, Imagawa S, Maeshima M (2001) Specificity of the accumulation of mRNAs and proteins of the plasma membrane and tonoplast aquaporins in radish organs. Planta 212: 294-304

Suga S, Murai M, Kuwagata T, Maeshima M (2003) Differences in aquaporin levels among cell types of radish and measurement of osmotic water permeability of individual protoplasts. Plant
Cell Physiol 44: 277-286

Tanaka I, Kitazume C, Ito M (1987) The isolation and culture of lily pollen protoplasts. Plant Sci 50: 205-211

Yoshida S, Uemura M, Niki T, Sakai A, Gusta LV (1983) Partition of membrane particles in aqueous two-polymer phase system and its practical use for purification of plasma membranes from plants. Plant Physiol 72: 105-114

Zelazny E, Borst JW, Muylaert M, Batoko H, Hemminga MA, Chaumont F (2007) FRET imaging in living maize cells reveals that plasma membrane aquaporins interact to regulate their subcellular localization. Proc Natl Acad Sci USA 104: 12359-12364 